Open Access

\title{
Biologically active, high levels of interleukin-22 inhibit hepatic gluconeogenesis but do not affect obesity and its metabolic consequences
}

Ogyi Park', Sung Hwan Ki ${ }^{1,3}$, Mingjiang Xu' ${ }^{1}$, Hua Wang ${ }^{1}$, Dechun Feng ${ }^{1}$, Joseph Tam ${ }^{2,4}$, Douglas Osei-Hyiaman², George Kunos ${ }^{2}$ and Bin Gao ${ }^{1 *}$

\begin{abstract}
Background: Interleukin-22 (IL-22), a cytokine with important functions in anti-microbial defense and tissue repair, has been recently suggested to have beneficial effects in obesity and metabolic syndrome in some but not in other studies. Here, we re-examined the effects of IL-22 on obesity, insulin resistance, and hepatic glucose metabolism.

Results: Genetic deletion of IL-22 did not affect high-fat-diet (HFD)-induced obesity and insulin resistance. IL-22 transgenic mice with relatively high levels of circulating $\mathrm{IL}-22(\sim 600 \mathrm{pg} / \mathrm{ml})$ were completely resistant to Concanavalin A-induced liver injury but developed the same degree of high fat diet (HFD)-induced obesity, insulin resistance, and fatty liver as the wild-type littermate controls. Similarly, chronic treatment with recombinant mouse IL-22 (rmlL-22) protein did not affect HFD-induced obesity and the associated metabolic syndrome. In vivo treatment with a single dose of rmlL-22 downregulated the hepatic expression of gluconeogenic genes and subsequently inhibited hepatic gluconeogenesis and reduced blood glucose levels both in HFD-fed and streptozotocin (STZ)-treated mice without affecting insulin production. In vitro exposure of mouse primary hepatocytes to IL-22 suppressed glucose production and the expression of gluconeogenic genes. These inhibitory effects were partially reversed by blocking STAT3 or the AMPK signaling pathway.

Conclusion: Biologically active, high levels of IL-22 do not affect obesity and the associated metabolic syndrome. Acute treatment with IL-22 inhibits hepatic gluconeogenesis, which is mediated via the activation of STAT3 and AMPK in hepatocytes.
\end{abstract}

Keywords: Obesity, Insulin resistance, Hyperglycemia, Cytokine, Liver

Interleukin-22 (IL-22) is probably the only cytokine that is produced by immune cells but does not directly target immune cells due to lack of IL-22 receptor 1 (IL-22R1) expression on these cells [1-6]. Instead, IL-22 mainly targets epithelial cells which express high levels of IL-22R1 [1-6]. Several types of immune cells have been reported to produce IL-22. These include Th17 cells, Th22 cells, activated NK and NKT cells and others [1-6]. The action of IL-22 is mediated via its binding to IL-10R2 and IL-22R1, followed by the activation of signal transducer and activator of transcription 3 (STAT3) and, to a

\footnotetext{
* Correspondence: bgao@mail.nih.gov

'Laboratory of Liver Diseases, NIAAA/NIH, 5625 Fishers Lane, Bethesda, MD 20892, USA

Full list of author information is available at the end of the article
}

lesser extent, activation of additional signaling pathways such as STAT1, STAT5, AKT, ERK, etc. [1-6]. IL-10R2 is ubiquitously expressed while IL-22R1 is expressed exclusively in epithelial cells (eg. hepatocytes), hepatic stellate cells [HSCs], and fibroblasts [1-7]. Accumulating evidence suggests that IL-22 plays a critical role in antimicrobial defense and tissue repair in various organs [1-6]. In the liver, through its action on hepatocytes, IL-22 has been shown to act as a hepatoprotective factor that protects against liver injury, fibrosis and steatosis via the activation of STAT3 in a variety of rodent models and patients [7-13]. IL-22 does not initiate liver cancer development but can promote proliferation of existing liver tumor cells via the activation of STAT3 [14-16]. Although it does not target immune cells, IL-22 may indirectly 
promote liver inflammation in diseased liver, such as in chronic viral hepatitis [17].

Numerous recent studies suggest that IL-22 modulates obesity and its metabolic consequences, but the results are inconsistent [18-24]. In an early study, injection of mice with adenovirus expressing IL-22, which produced super high levels of circulating IL-22, induced marked body weight loss and thymic atrophy in lean mice [18]. We also observed that IL-22 transgenic mice expressing super high levels of IL-22 (4000-7000 pg/ml) had much lower lean body weight [14]. It should be noted that serum IL-22 levels in healthy individuals and patients with various types of diseases rarely exceed $200 \mathrm{pg} / \mathrm{ml}$ [15, 25, 26]. Recently, Wang et al. [19] reported that treatment with high doses of long half-life IL-22Fc protein $\left(50-100 \mu \mathrm{g} /\right.$ mouse, half-life $\mathrm{t}_{1 / 2}=3.02$ days, twice a week for 4 weeks) reduced body weight and ameliorated hyperglycemia and insulin resistance in obese, leptin receptor-deficient mice and mice fed a high-fat diet (HFD). Interestingly, Hasnain et al. [20] reported that chronic treatment with low doses of short half-life recombinant mouse IL-22 protein (rmIL-22)(20 ng/g or $100 \mathrm{ng} / \mathrm{g}$, twice a week for 4 weeks) reduced body weight and alleviated metabolic complications caused by HFD in mice. However, Yang et al. [21] found that chronic treatment with rmIL-22 (300 ng/g, daily for 36 days) ameliorated fatty liver but did not affect body weight and metabolic parameters in HFD-fed mice. In contrast, Upadhyay et al. [22] observed that overexpression of IL-22 via hydrodynamic injection restored normal body weight and adiposity in lymphotoxin $\beta$ receptor knockout mice. In addition, several studies suggested that T cell-derived IL-22 enhances IL- $1 \beta$-mediated inflammation in human adipose tissue and reduces insulin sensitivity in human hepatocytes, promoting obesity and diabetes [23, 27].

In the current study, we analyzed the effects of endogenous IL-22 and chronic treatment with rmIL-22 as well as genetic overexpression of IL-22 on HFD-induced obesity and metabolic syndrome. Our results indicate that high circulating levels of transgenically expressed IL-22, chronic treatment with rmIL-22, or deficiency in endogenous IL-22 do not affect HFD-induced obesity and its metabolic consequences in mice. The effect of IL-22 on glucose metabolism in hepatocytes were also examined.

\section{Results}

Endogenous IL-22 does not play a role in regulating obesity, insulin resistance, and fatty liver disease induced by 10 weeks of HFD feeding

It was reported that basal serum levels of IL-22 were approximately $20 \mathrm{pg} / \mathrm{ml}$ in control diet-fed mice, and were decreased to $5 \mathrm{pg} / \mathrm{ml}$ in HFD-fed mice [28]. However, a recent study reported that serum levels of IL-22 were markedly increased after HFD diet feeding to approximately $1000 \mathrm{pg} / \mathrm{ml}$ compared to approximately $100 \mathrm{pg} / \mathrm{ml}$ in chow-fed mice (Extended data Fig. 1 in reference [19]). Here we found that serum IL-22 levels were relatively low $(<20 \mathrm{pg} / \mathrm{ml})$ in control- or HFD-fed mice with lower levels in HFD-fed mice than those in control-fed mice, while high levels of serum IL-22 were detected in IL-22TG6 mice $(\sim 600 \mathrm{pg} / \mathrm{ml})$ (Fig. 1a). HFD feeding did not affect serum IL-22 levels in IL-22TG6 mice.

To determine the role of IL-22 in obesity and insulin resistance, we bred IL-22 $2^{+/-}$heterozygous mice to generate wild-type littermates and IL-22KO mice. These mice were fed a HFD or control diet for 10 weeks. As illustrated in Fig. 1b, both WT and IL-22KO mice had similar body weight gain, glucose intolerance, and insulin resistance after 10 weeks of HFD feeding. In addition, hepatic steatosis as determined by $H \& E$ staining and measurement of hepatic triglyceride levels was comparable between HFD-fed WT and IL-22KO mice (data not shown). This suggests that very low levels of endogenous IL-22 do not contribute to the pathogenesis of obesity, insulin resistance, and fatty liver disease in the 10-week HFD feeding model.

\section{Liver-specific IL-22TG6 mice with relatively high levels of circulating IL-22 ( $600 \mathrm{pg} / \mathrm{ml})$ are resistant to concanavalin A-induced liver injury but develop the same degree of HFD-induced obesity, insulin resistance, and fatty liver as wild-type littermates}

The role of IL-22 in regulating obesity and metabolic syndrome was further examined in IL-22 transgenic mice, in which IL-22 expression is controlled by albumin promoter and enhancer [14, 29]. We obtained four lines of IL-22TG mice, including three lines (IL-22TG8, TG9, and TG15) that have super high levels of circulating IL-22 (4000-7000 pg/ml) and one line (IL-22TG6) that has relatively high levels of circulating IL-22 $(\sim 600 \mathrm{pg} / \mathrm{ml})$ (Fig. 1a) $[14,29]$. We have previously demonstrated that the three lines with super high levels of IL-22 were completely resistant to Con A-induced liver injury [14]. Here we report that IL-22TG6 mice were also completely resistant to Concanavalin A-induced liver injury, as demonstrated by the marked elevation of serum ALT and AST in WT mice but not in IL-22TG mice (Fig. 2a). Liver histology analysis revealed that WT mice had massive necrosis while IL-22TG6 mice only had a few of small necrotic areas (Fig. 2b). This suggests that high levels of IL-22 in IL-22TG6 are biologically active.

We have previously found that the IL-22TG8, TG9, and TG15 mice had much lower lean body weight [14], suggesting that super high IL-22 levels may cause cachexia. The IL-22TG6 with relatively high levels of circulating IL-22 had the same lean body weight as the wild-type 
A

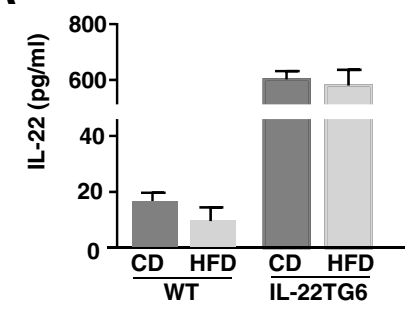

C

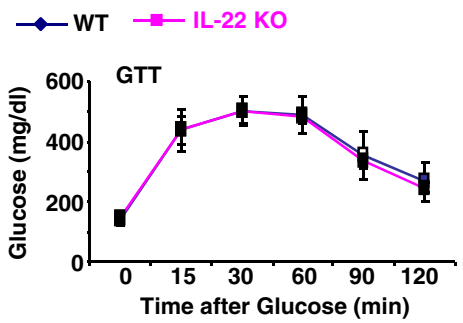

B
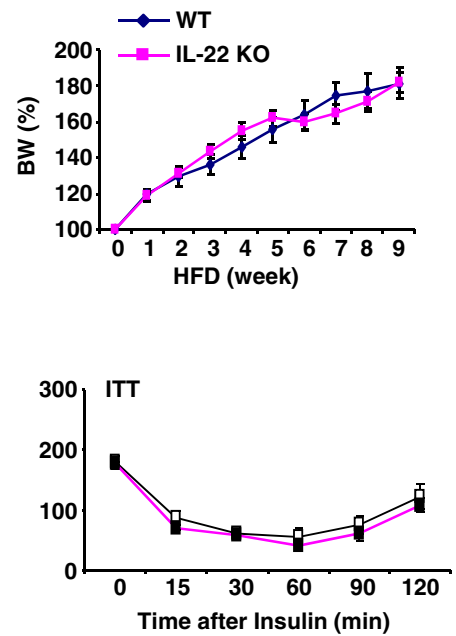

Fig. 1 Endogenous IL-22 does not play a role in the development of obesity and insulin resistance induced by a HFD. a Two-month old IL-22TG6 mice and their littermate controls were fed a HFD or CD for 10 weeks. Serum IL-22 levels were measured. b, c Two-month old IL-22KO mice and their littermate controls were fed a HFD or CD for 10 weeks. Body weight was counted weekly (panel b); GTT and ITT were examined after 10 weeks feeding (panel c). CD; control diet; HFD: high-fat diet. Values represent the mean \pm SEM $(n=6-12)$

littermate controls (Fig. 3a). Therefore, in the current study, HFD-induced obesity and its metabolic consequences were only examined in IL-22TG6 mice. After HFD feeding, IL-22TG6 mice gained comparable body weight and had the same total adiposity as WT littermate controls (Fig. 3a-b). Glucose tolerance test (GTT) and insulin tolerance test (ITT) analyses revealed that there were no differences in glucose tolerance or insulin sensitivity between CD- or HFD-fed WT and IL-22TG6 mice (Fig. 3c-d). Moreover, serum ALT levels, hepatic TG levels, and hepatic steatosis were also comparable in HFD-fed WT and IL-22TG6 mice (Fig. 3e-f).

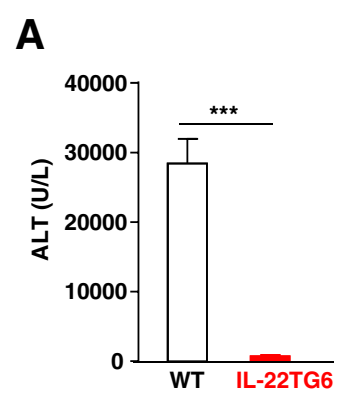

B

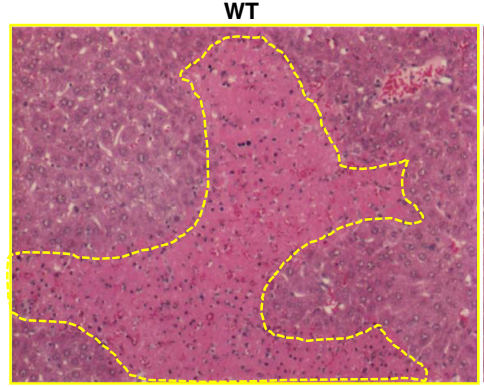

IL-22TG6

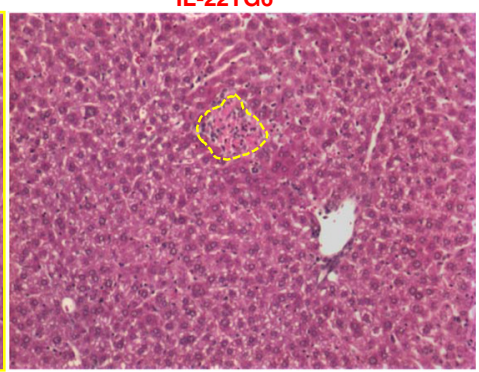

Fig. $2 \mathrm{IL}-22 \mathrm{TG} 6$ mice with high levels of circulating IL-22 ( 600 pm/ml) are resistant to Con A-induced liver injury. WT and IL-22TG mice were injected with Con A $(15 \mu \mathrm{g} / \mathrm{g})$ for $24 \mathrm{~h}$. a Serum ALT and AST levels were measured. b Representative H\&E staining of liver tissues from mice treated with Con A for $24 \mathrm{~h}$. Necrotic areas are indicated by dot circles. Values represent the mean \pm SEM $(n=10-14)$. ${ }^{* * *} P<0.001$ 

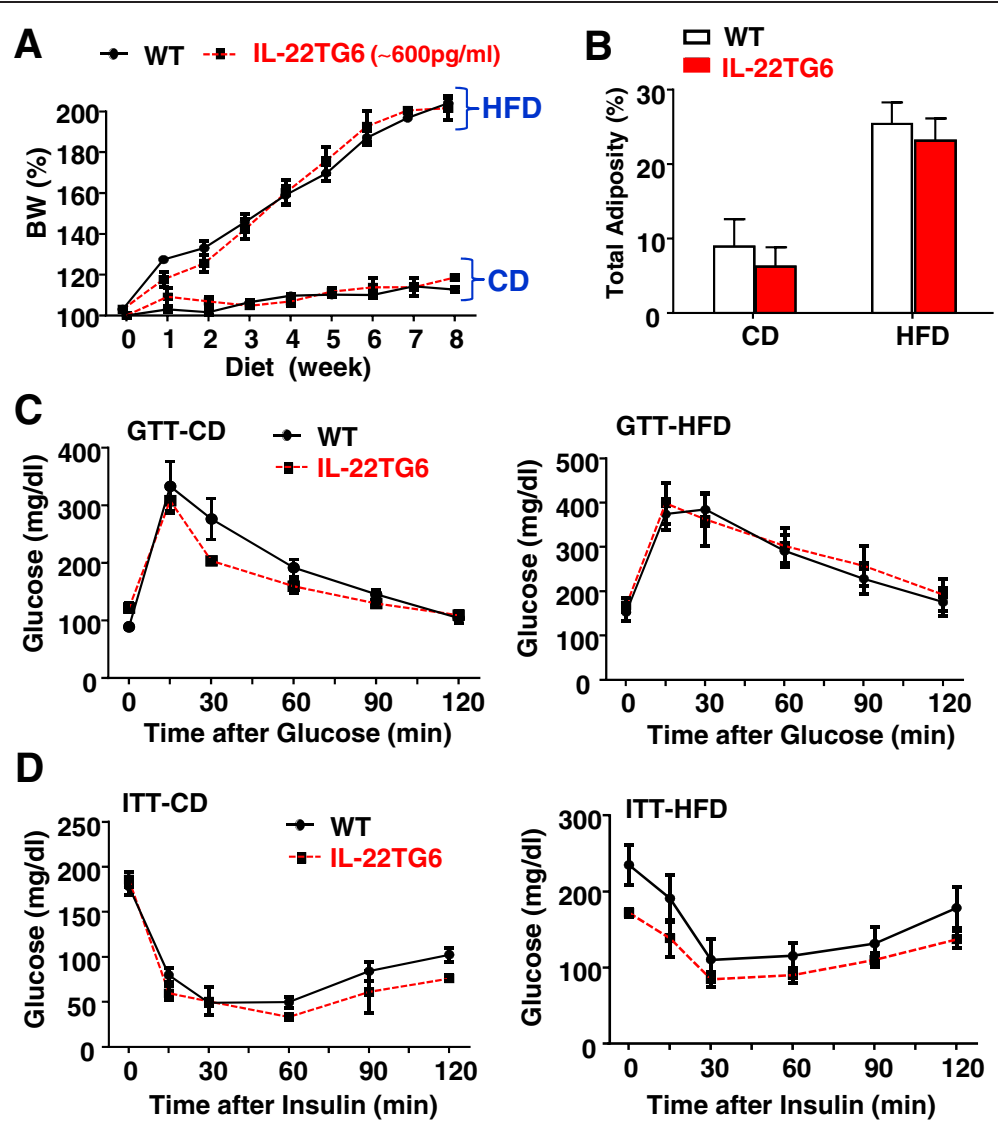

$\mathbf{E}$
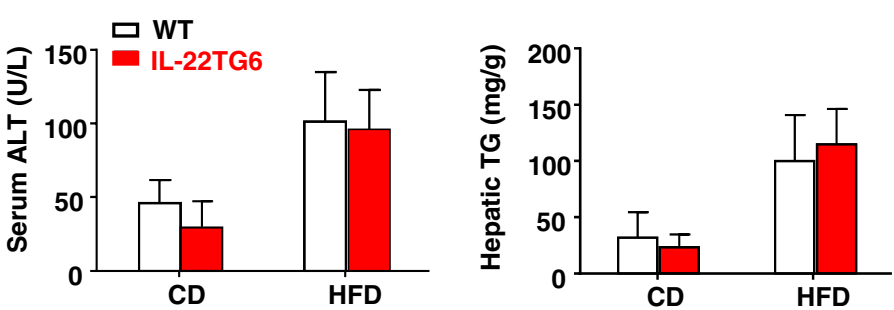

$\mathbf{F}$
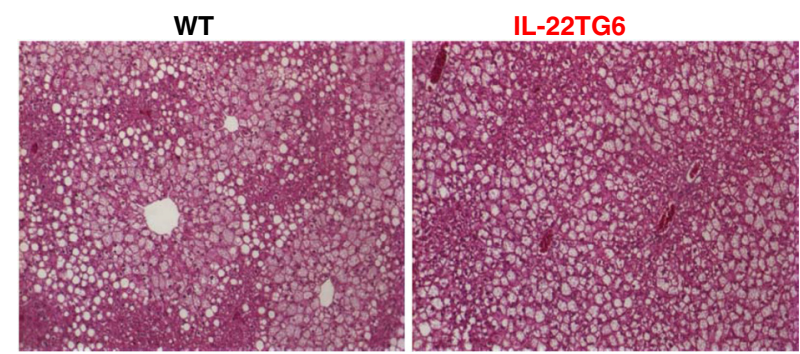

Fig. 3 IL-22TG6 mice with high levels of circulating IL-22 ( 600 pg/ml) develop the same degree of HFD-induced obesity, insulin resistance, and fatty liver as wild-type littermates. Mice were fed a CD or HFD for 8 weeks. a Body weights were measured. b Total adiposity. c Glucose tolerance test (GTT). d Insulin resistance test (ITT). e Serum ALT and hepatic TG levels were measured. $\mathbf{f}$ Representative H\&E staining of liver tissues from 8-week HFD fed mice. Values represent the mean \pm SEM $(n=6-14)$

Chronic treatment with recombinant mouse IL-22 (rmlL-22) protein does not ameliorate HFD-induced obesity and the associated metabolic syndrome

In an early study, chronic treatment with rmIL-22 $(300 \mathrm{ng} / \mathrm{g}$, daily injection) (Generon Corporation, Shanghai, China) for 36 days ameliorated fatty liver but did not affect body weight, fasting glucose, and fasting insulin levels in HFD-fed mice [21], while a recent study reported that chronic treatment with low doses of rmIL-22 (R\&D systems) (20 ng/g or $100 \mathrm{ng} / \mathrm{g}$ body weight, twice a week) 
ameliorated obesity and metabolic syndrome in HFD-fed mice [20]. One of the reasons for this discrepancy might be the different sources of rmIL-22 were used.

To further clarify this discrepancy, we treated HFD-fed mice with rmIL-22 (R\&D system) (20 ng/g body weight, twice a week) for 4 weeks. Our results revealed that such treatment did not affect body weight and fasting glucose levels (Fig. 4a-b) and insulin and glucose intolerance (data not shown).

\section{Administration of a single dose of the rmIL-22 protein reduces blood glucose levels in HFD-fed and streptozocin (STZ)-treated mice without affecting blood insulin levels: IL-22 activates STAT3 in acinar cells but not in islets in the pancreas}

To explore whether pharmacologic doses of rmIL-22 have any acute beneficial metabolic effects, we treated mice with a single dose of the rmIL-22 protein. Administration of a single dose of rmIL-22 (1000 ng/g body weight) did not significantly affect body weight in mice fed a HFD or CD (data not shown). However, injection of a single dose of rmIL-22 significantly decreased fasting blood glucose levels in mice fed a HFD and decreased plasma glucose levels in mice fed a CD to a lesser extent (Fig. 5a).

Next, we tested whether IL-22 also reduces blood glucose levels in a model of type I diabetes induced via STZ injection. Injection of STZ caused pancreatic islet damage (data not shown) and decreased serum insulin levels in WT mice (Fig. 5b). Similar pancreatic islet damage was observed in STZ mice treated with or without IL-22 treatment (data not shown). Moreover, as illustrated in Fig. 5b-c, injection of a single dose of rmIL-22 did not affect the pancreas weight nor serum insulin levels in both vehicle- or STZ-treated groups but markedly reduced blood glucose levels in STZ-treated mice.

We and others previously reported that IL-22 treatment protects against cerulean-induced pancreatitis in mice by targeting pancreatic acinar cells [30, 31]. Interestingly, a recent study reported that IL-22 can directly target mouse and human pancreatic islet beta cells [20]. However, immunohistochemistry analyses revealed strong pSTAT3 staining in acinar cells but not in islets in the pancreas from IL-22-treated mice (Fig. 5d).

\section{IL-22 inhibits hepatic gluconeogenesis without affecting glucose uptake}

To explore the mechanisms by which IL-22 reduces blood glucose levels in HFD-fed mice, we performed an in vivo glucose turn-over assay and pyruvate tolerance test (PTT), an assay to determine hepatic gluconeogenesis in vivo. HFD-fed mice were injected with IL-22 adenovirus or control adenovirus prior to the glucose turn-over assay. Injection of Ad-IL-22 resulted in significant elevation of circulating IL-22 ( $5000 \mathrm{pg} / \mathrm{ml})$, and this elevation lasted for more than two weeks [10]. As illustrated in Fig. 6a, the glucose turn-over rate and blood glucose levels were markedly lower in ad-IL-22-treated mice compared with ad-vector-treated mice. Fig. $6 \mathrm{~b}$ shows that blood glucose levels were significantly elevated after injection of pyruvate in ad-vector-treated mice; however, such elevation was not observed in ad-IL-22 injected mice, suggesting that injection of ad-IL-22 blocks hepatic gluconeogenesis. Indeed, expression of gluconeogenic genes, including G6Pase, PEPCK, and TORC2 (also known as CRTC2), was markedly reduced in ad-IL-22-treated mice compared with ad-vector-treated mice (Fig. 6c). In addition, administration of a single dose of rmIL-22 markedly suppressed hepatic expression of gluconeogenic genes (Fig. 6d).

Glucose uptake experiments showed that IL-22 treatment did not affect glucose uptake in the liver, muscle, and WAT tissues (Fig. 6e). There was a trend towards an increase in BAT in mice treated with rmIL-22, but it did not reach statistical significance.

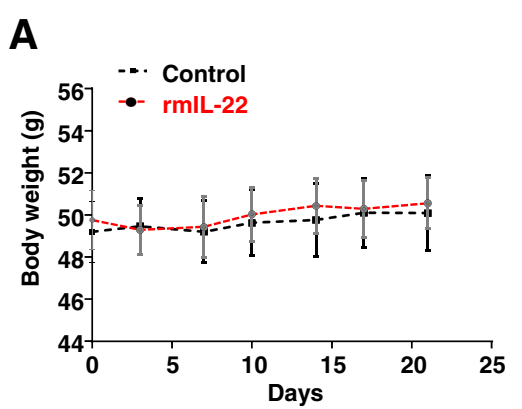

B

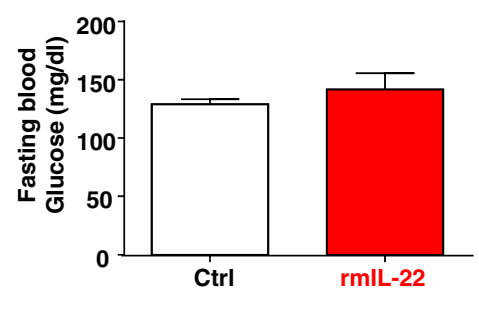

Fig. 4 Chronic administration of rmlL-22 protein does not affect body weight and insulin resistance in HFD-fed mice. Mice were fed a HFD for 5 months and then injected with $\mathrm{rmlL}-22$ ( $20 \mathrm{ng} / \mathrm{g}$ body weight, i.p. injection, twice a week) or saline for an additional four weeks. a Body weights were measured. $\mathbf{b}$ Fasting blood glucose levels. Values represent the mean $\pm \operatorname{SEM}(n=8)$ 


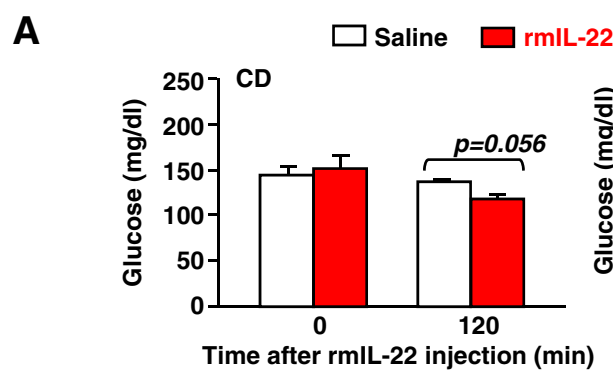

B

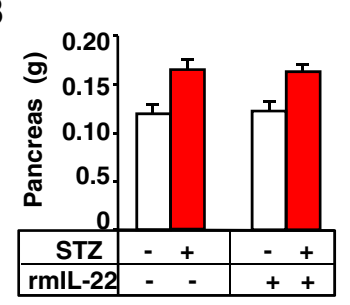

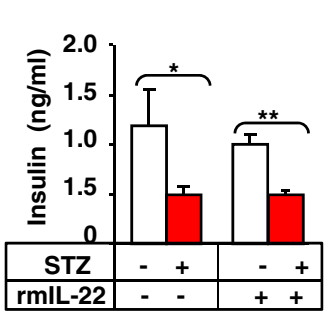

C

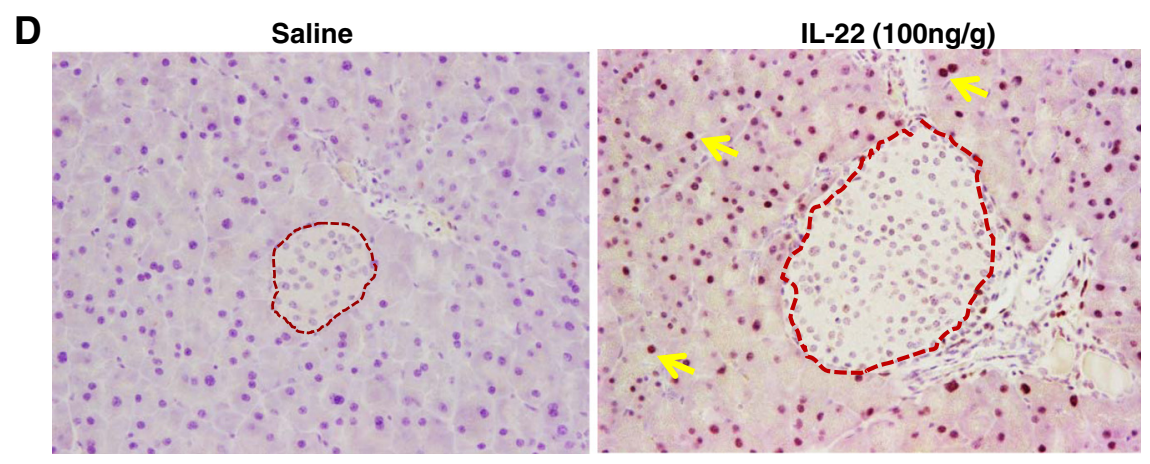

Fig. 5 Injection of a single dose of recombinant mouse IL-22 ( $\mathrm{rmlL}-22$ ) protein reduces blood glucose levels in HFD- and streptozotocin (STZ)-treated mice. a Mice were fed a HFD for 8 weeks and then injected with saline or rmlL-22 for $2 \mathrm{~h}$. Blood glucose levels were detected 120 min post IL-22 injection. b, c C57BL/6 mice were injected with STZ for 5 consecutive days. Twenty eight days later, mice were injected with saline or rmIL-22, and sacrificed $2 \mathrm{~h}$ later. Pancreas weights and insulin levels were measured (panel b). Glucose levels were measured at various time points post rmlL-22 injection (panel $\mathbf{c}$ ). Values represent the mean \pm SEM $(n=10)$. ${ }^{*} P<0.05$ and ${ }^{* *} P<0.01$ compared with the corresponding saline treated groups. d C57BL/6 mice were treated with rmlL-22 for $2 \mathrm{~h}$, pancreas tissues were collected for immunostaining with anti-pSTAT3 antibody. Representative positive pSTAT3 nuclei in acinar cells are indicated by yellow arrows but not in islets (indicated by dotted lines)

STAT3 and AMPK but not PI3/AKT contribute to IL-22 inhibition of hepatic gluconeogenesis in vitro

To further understand the mechanisms underlying IL-22mediated inhibition of hepatic gluconeogenesis, we examined the effects of IL-22 signaling pathways on hepatic gluconeogenesis and gluconeogenic genes in primary mouse hepatocytes. As shown in Figs. 7a-b, treatment with rmIL-22 predominantly induced the phosphorylation and activation of STAT3 and to a lesser extent induced pAKT and pAMPK activation in primary mouse hepatocytes. Compared to insulin stimulation, rmIL-22 induced much weaker pAKT activation in primary mouse hepatocytes. In addition, rmIL-22 treatment did not further enhance insulin activation of pAKT (data not shown).

Next, we investigated whether these signaling pathways contributed to the IL-22-mediated inhibition of glucose production in primary hepatocytes. As shown in
Fig. 7c, treatment with rmIL-22 decreased basal glucose production, and expression of gluconeogeneic genes, including PEPCK, G6Pase, and PGC-1 $\alpha$, in WT hepatocytes. IL-22 inhibition of glucose production was completely abolished, whereas IL-22 inhibition of these genes was partially diminished in STAT3 knockout hepatocytes. Interestingly, treatment with AMPK inhibitor but not with the PI3 kinase inhibitor LY294002 partially diminished the IL-22 inhibition of glucose production and G6Pase gene expression in WT hepatocytes.

Similarly, treatment with rmIL-22 also decreased Bt2-cAMP-induced glucose production in WT mouse hepatocytes but not in STAT3 knockout hepatocytes. Such inhibition was also partially diminished in AMPK inhibitor-treated hepatocytes but not in PI3 inhibitortreated hepatocytes (Fig. 7d). Treatment with rmIL-22 inhibited the Bt2-cAMP induction of PEPCK, G6Pase, 

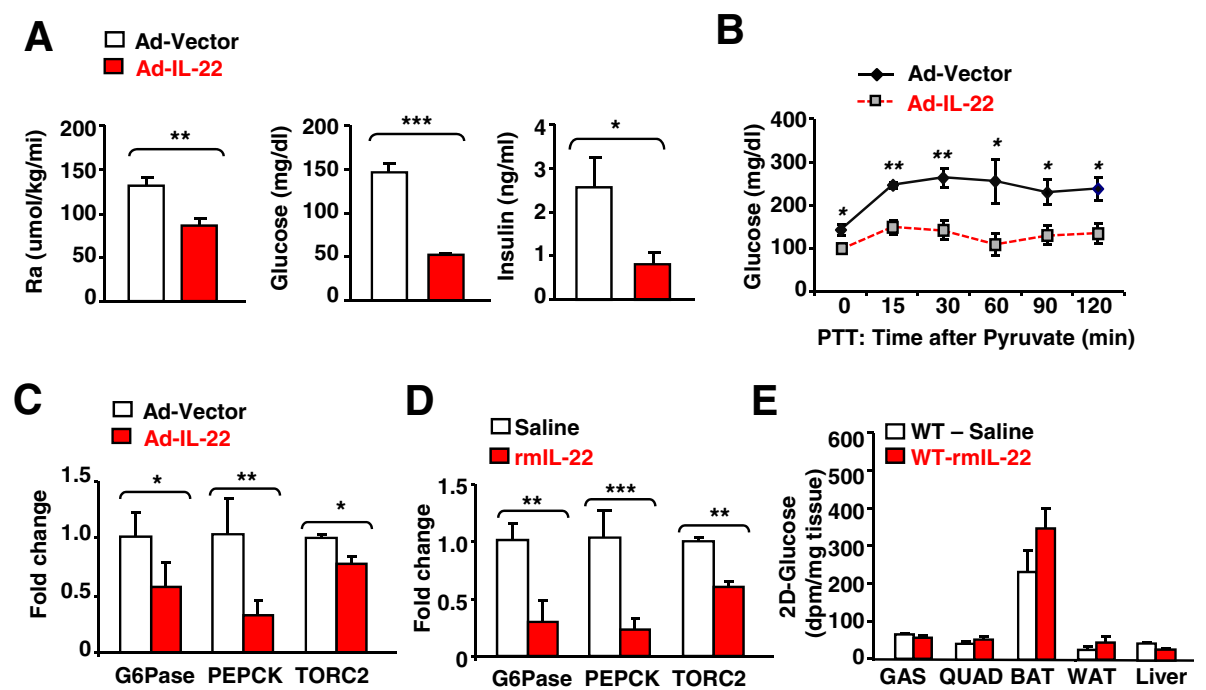

Fig. 6 IL-22 inhibits hepatic gluconeogenesis without affecting glucose uptake in vivo. a-c Mice were fed a HFD for 8 weeks and then injected with ad-vector or ad-IL-22 for 5 days. A glucose tracer assay in vivo was performed. Glucose turnover rates and plasma glucose levels are shown (panel a). A pyruvate tolerance test (PTT) was performed (panel b). Real-time PCR analyses of gluconeogenic genes (panel c). d-e C57BL/6 mice were fed a HFD for 8 weeks and then fasted for $4 \mathrm{~h}$, followed by treatment with saline or rmlL-22 (1 $\mu \mathrm{g} / \mathrm{g})$ for $2 \mathrm{~h}$. Real-time PCR analyses of gluconeogenic genes (panel d). Two-deoxyglucose uptake experiments in vivo were performed (panel e). Values represent the mean \pm SEM $(n=6-10)$. *P<0.05, ${ }^{* *} P<0.01$, and ${ }^{* *} P<0.001$ compared with the corresponding ad-IL-22-treated or rmIL-22-treated groups

and PGC- $1 \alpha$ expression in WT mice (Fig. $7 d$ ). rmIL-22 inhibition of PEPCK and G6Pase but not PGC-1 $\alpha$ was partially diminished in STAT3 knockout hepatocytes and AMPK inhibitor-treated hepatocytes (Fig. 7d).

\section{Discussion}

As mentioned in the introduction, the effects of IL-22 on obesity and metabolic syndrome are very controversial. Here, we provide evidence that endogenous IL-22 or biologically active, high circulating levels of IL-22 do not affect HFD-induced obesity and its metabolic consequences, although IL-22 is able to inhibit hepatic gluconeogenesis in hepatocytes.

\section{Endogenous IL-22 does not play a role in modulating HFD-induced metabolic syndrome}

It has been reported that serum levels of IL-22 in mice are decreased by HFD feeding from approximately $20 \mathrm{pg} / \mathrm{ml}$ in lean controls to approximately $5 \mathrm{pg} / \mathrm{ml}$ [28]. In our study, serum levels of IL-22 were also relatively low $(<20 \mathrm{pg} / \mathrm{ml})$ in both CD and HFD-fed mice (Fig. 1a). In addition, we found that IL-22TG6 mice, which have high circulating levels of IL-22 $(\sim 600 \mathrm{pg} / \mathrm{ml})$, develop the same degree of HFD-induced obesity and its metabolic consequences as wild-type littermates. Thus, the very low levels of endogenous IL-22 are unlikely to contribute to the pathogenesis of HFD-induced obesity and its metabolic sequelae. This notion is further supported by our finding that HFD feeding induced the same degree of body weight gain, obesity, and insulin resistance in WT littermates and IL-22KO mice. Recently, Wang et al. [19] also reported that HFD feeding induced comparable levels of obesity in IL-22KO mice and WT mice, whereas IL-22R1KO mice were more susceptible to HFD-induced obesity and insulin resistance. Because IL-22R1 can combine not only with IL-10R2 to act as a functional IL-22R complex but can also interact with IL-20R2 to form a receptor for IL-20 and IL-24 [32], it is likely that IL-22R1 ligands other than IL-22 may play a role in ameliorating HFD-induced metabolic syndrome.

\section{Biologically active, high levels of IL-22 do not modulate} HFD-induced obesity and its metabolic consequences Super high levels of circulating IL-22 (4000-7000 pg/ml) in IL-22TG8 mice or in mice treated with ad-IL-22 caused marked body weight loss in lean mice [14, 18], suggesting that super high levels of IL-22 induce cachexia. At present, the mechanisms underlying IL-22-mediated cachexia remain unclear. It has been well-documented that a wide variety of cytokines can induce cachexia after prolonged production via multiple mechanisms, and these cytokines include TNF- $\alpha$, IL-6, leukemia inhibitory factor (LIF), ciliary neurotrophic factor (CNTF) and interferon- $\gamma$ $($ IFN- $\gamma)$ [33]. It is likely that super high levels of IL-22 promote cachexia by using mechanisms similar to those used by these cytokines such as induction of strong acute phase response and subsequent chronic inflammation [18, 33]. Although it was reported that IL-22 can indirectly induce inflammation in chronic liver disease [17], hepatic and serum levels of IL- 6 , TNF- $\alpha$, IL-1 $\beta$ were not elevated in 

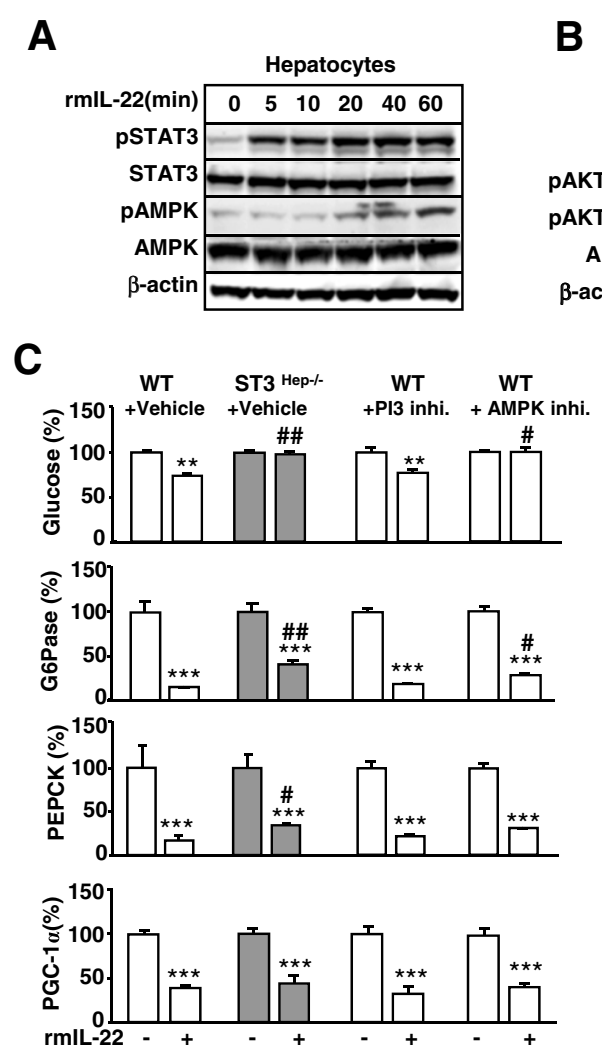

B

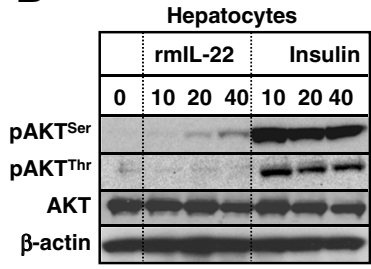

D

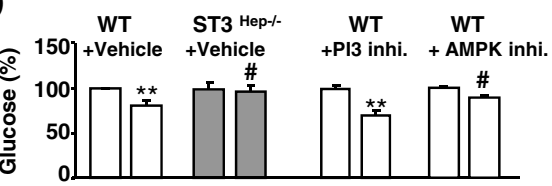

ऽ 150
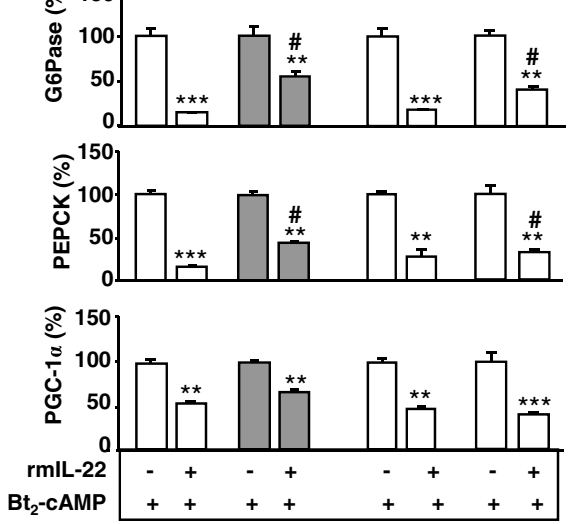

Fig. 7 Treatment with rmlL-22 protein inhibits gluconeogenesis in primary mouse hepatocytes via STAT3- and AMPK-dependent mechanisms. a Western blot analyses of IL-22-treated primary mouse hepatocytes. b Western blot analyses of IL-22- or insulin-treated hepatocytes. c Primary wild-type mouse hepatocytes with pre-treated PI3K or AMPK inhibitors, followed by IL-22 treatment. Primary STAT3KO mouse hepatocytes were also treated with IL-22. $\mathbf{d}$ The same experiments as those in panel C except all cells were pre-treated with Bt2-cAMP. In panels $\mathbf{c}$ and $\mathbf{d}$, glucose production and gene expression were analyzed and normalized to $100 \%$ in hepatocytes without IL-22 treatment in each group. Values represent the mean $\pm \operatorname{SEM}(n=4) .{ }^{*} P<0.05,{ }^{* *} P<0.01$, and ${ }^{* *} P<0.001$ compared with the corresponding hepatocytes without rmlL-22 treatment. ${ }^{*} P<0.05$ and ${ }^{\# \# P}<0.01$ compared with the corresponding hepatocytes from vehicle + WT mice with rmIL-22 treatment

mice treated with high doses of Ad-IL-22 [18] or in IL-22TG8 mice with super high levels of circulating IL-22 $(\sim 6000 \mathrm{pg} / \mathrm{ml})$ (Park et al. unpublished data). This suggests that the cachectic effect of super high levels of IL-22 is not due to upregulation of the cachectic factors such as IL- 6 and TNF- $\alpha$.

Recently, Wang et al. [19] reported that chronic treatment of mice with high doses of IL-22Fc protein (50-100 $\mu \mathrm{g} /$ mouse, twice a week) for 6 weeks markedly reduced the body weights of HFD-fed mice. IL-22Fc has a long half-life $\left(t_{1 / 2}=3.02\right.$ days $)$ and administration of high doses of IL-22Fc may result in sustained high levels of IL-22 in vivo. Although Wang et al. reported that IL-22Fc treatment reduced fat pad size in HFD-fed mice, they did not analyze body composition, nor did they examine the effect of IL-22Fc treatment on body weight in lean mice, so we can't rule out the possibility that the weight-reducing effect of IL-22Fc treatment in HFD-fed mice was due to cachexia caused by super high levels of IL-22.
In an early study, chronic treatment with rmIL-22 (300 ng/g, daily for 36 days) improved fatty liver but did not affect body weight and the associated metabolic syndrome in HFD-fed mice [21]. This is consistent with our findings that relatively high levels of IL-22 in IL-22TG6 mice do not affect obesity. Lack of the anti-obesity effects of high levels of circulating IL-22 (600 pg/ml) in IL-22TG6 mice was very unlikely due to developing hepatic IL-22 resistance because IL-22TG6 mice were completely resistant to Con A-induced liver injury. Surprisingly, a recent study reported that chronic treatment with low doses of rmIL-22 markedly ameliorated obesity and metabolic syndrome in HFD-fed mice [20]; however, these results could not be reproduced in the present study.

\section{IL-22 regulates lipid and glucose metabolism}

Previous studies have revealed that IL-22 ameliorates fatty liver disease by downregulating hepatic expression of several lipogenic genes $[10,21]$. In the current study, we have demonstrated that IL-22 also regulates glucose 
metabolism via the inhibition of hepatic gluconeogenesis. First, injection of adeno-IL-22 inhibited glucose turn-over rate and gluconeogenesis in the liver. Second, treatment with rmIL-22 inhibited glucose production in primary mouse hepatocytes. Finally, treatment with IL-22 markedly reduced the expression of gluconeogenic genes, including PEPCK and G6Pase, in the liver in vivo and in hepatocytes in vitro. The STAT3, which is the major downstream of IL-22 signaling pathway, has been shown to inhibit hepatic lipogenesis and gluconeogenesis [34], which is in line with the effect of IL-22 on lipid and glucose metabolism in hepatocytes. In addition to the predominant activation of STAT3 in hepatocytes, IL-22 also activates, to a lesser extent, many other signaling pathways including AKT (as shown in Fig. 7 in this paper, and references [1-3]). Both STAT3 and AKT are known to inhibit hepatic gluconeogenesis [34, 35], but our results suggest that activation of STAT3 and not that of AKT is involved in the IL-22 suppression of hepatic gluconeogenesis. In vitro treatment with rmIL-22 inhibited glucose production in primary WT mouse hepatocytes but not in STAT3 knockout hepatocytes. Interestingly, although the effects of IL-22 on glucose production were completely absent in STAT3 knockout hepatocytes, its effect on gluconeogenic gene expression was only partially reduced, suggesting the involvement of additional signaling pathways in this latter effects. Despite an important role for AKT in inhibiting hepatic gluconeogenesis [35], inhibition of PI3/AKT by the PI3 kinase inhibitor LY294002 did not alter the effects of IL-22 on glucose production and gluconeogenic gene expression. The lack of a role for AKT in IL-22 inhibition of gluconeogenesis may be related to the fact that IL-22 only induced very weak AKT activation in hepatocytes.

In this study, we have demonstrated for the first time that IL-22 treatment activates AMPK in hepatocytes. AMPK has been shown to play an important role in inhibiting hepatic gluconeogenesis [36]. Our findings that the AMPK inhibitor compound $\mathrm{C}$ abolished the IL-22-mediated inhibition of glucose production and gluconeogenic gene expression suggest that the activation of AMPK is also involved in the IL-22-mediated inhibition of gluconeogenesis in hepatocytes.

\section{IL-22 treatment does not protect against STZ-induced islet damage and insulin reduction in mice}

It is well documented that IL-22R1 is primarily expressed in epithelial cells, such as acinar cells in the pancreas [3, 30, 31]. Interestingly, Hasanin et al. recently reported that pancreatic beta cells express IL-22R1, and IL-22 administration suppresses ER stress and inflammation and promotes insulin secretion in beta cells [20]. However, several lines of evidence argue against this notion. First, blood insulin levels were lower in IL-22TG8 mice than in WT mice under HFD or normal chow feeding (Park et al. unpublished data). Second, administration of STZ causes beta cell damage and reduces insulin production, which was not averted by administration of rmIL-22. Third, STAT3, which is a major downstream signaling molecule activated by IL-22, mediates the cytoprotective functions of IL-22 [3]. Injection of IL-22 resulted in strong pSTAT3 activation in acinar cells but not in beta cells. This activation in acinar cells contributes to the protective effects exerted by IL-22 on acinar cells and pancreatitis [30, 31]. Although we did not detect STAT3 activation in beta cells from IL-22-treated mice, we can't rule out that IL-22 may stimulate the STAT3 pathway at low levels, which may still have a beneficial effect under stress conditions, or the unlikely possibility that IL-22 activates other signaling pathways in beta cells. Further studies are needed to clarify the functions of IL-22 in beta cells in the pancreas.

In summary, our findings suggest that biologically active, high levels of IL-22 do not affect obesity and its metabolic consequence. Super high levels of IL-22 may cause cachexia and subsequently body weight loss. IL-22 inhibits hepatic gluconeogenesis via the activation of STAT3 and AMPK without affecting insulin production.

\section{Materials and methods \\ Materials}

Recombinant murine IL-22 protein (rmIL-22) was purchased from R\&D system (Minneapolis, MN). All of the antibodies used for Western blot analysis were purchased from Cell Signaling (Danvers, MA).

\section{Mice}

Eight- to ten-week-old male C57BL/6 N mice were purchased from the National Cancer Institute. Liver-specific IL-22 transgenic mice line 6 (IL-22TG6) on a C57BL/6 N background were generated as described previously [29]. IL-22TG-6 mice had relatively high serum levels of IL-22 ( $\sim 600 \mathrm{pg} / \mathrm{ml})$. IL-22 knockout out mice on a C57BL/6 background were kindly provided by Dr. Wenjun Ouyang (Genentech, San Francisco, CA, USA). All animal study protocols were reviewed and approved by the Institutional Animal Care and Use Committee of the National Institute on Alcohol Abuse and Alcoholism, National Institutes of Health.

\section{Diet-induced obesity}

Eight- to ten-week-old male mice were fed either a control diet (CD) or a high-fat diet (HFD) for 8 to 12 weeks or 5 months. The HFD contained $34.0 \%$ fat $(60 \%$ of calories), $26.3 \%$ carbohydrates (20\% of calories), and $26.2 \%$ protein (20\% of calories) as well as fiber, vitamins, and minerals (D12492, Research Diets, New Brunswick, NJ, USA). The CD contained $4.3 \%$ fat (10 \% of calories) (D12450B, Research Diets). The mice had free access to 
food and water. After feeding for various time periods, mice were euthanized and the liver and adipose tissues (gonadal, retroperitoneal, and subcutaneous) were removed, weighed, and snap-frozen. The adiposity index was calculated as the combined adipose tissue weight/ carcass body weight $\times 100 \%$.

\section{Streptozotocin (STZ)-induced type I diabetes}

Mice received 5 daily consecutive injections of $50 \mathrm{mg} / \mathrm{kg}$ STZ (dissolved in citrate buffer, pH 4.5, i.p. injection) in a volume that did not exceed $50 \mu$ l. Blood glucose levels were assessed after the last injection and then assessed weakly for 4 weeks.

\section{Glucose tolerance test (GTT), pyruvate tolerance test (PTT), and insulin tolerance test (ITT)}

For the GTT, mice were fasted overnight, and tail vein blood was collected to measure glucose levels. The mice were then injected with glucose ( $2 \mathrm{~g} / \mathrm{kg}$ i.p.), followed by the collection of tail vein blood and measurement of blood glucose levels at various time points. Blood glucose concentrations were measured using a Glucometer Contour (Bayer HealthCare, Mishawaka, IN, USA). For the ITT, mice were fasted for $6 \mathrm{~h}$, and tail vein blood was collected to measure basal blood glucose levels. Mice were then injected with insulin $(0.75 \mathrm{U} / \mathrm{kg}$ i.p., Eli Lilly), and blood glucose levels were measured at various time points. For the PTT test, mice were fasted for $16 \mathrm{~h}$ and injected with pyruvate $(2 \mathrm{~g} / \mathrm{kg}$ i.p.), and blood glucose levels were measured at various time points.

\section{Endogenous glucose production measurement in vivo}

Basal glucose production was measured in restrained, conscious mice maintained on HFD for 8 weeks. Four days before the experiment, mice were anesthetized with $100 \mathrm{mg} / \mathrm{kg}$ ketamine and $10 \mathrm{mg} / \mathrm{kg}$ xylazine. A catheter was inserted through a lateral incision on the right side of the neck into the superior vena cava via the right internal jugular vein. The catheter was then sutured into place according to the protocol of MacLeod and Shapiro [37]. Experiments were started $3 \mathrm{~h}$ after fasting. The basal rates of glucose turnover were measured by continuous infusion of $\left[3-{ }^{3} \mathrm{H}\right]$ glucose $(2 \mu \mathrm{Ci}$ bolus, then $0.05 \mu \mathrm{Ci} / \mathrm{min}$ ) for $180 \mathrm{~min}$. Infusions were performed using microdialysis pumps (CMA 402/Microdialysis, Acton, MA, USA). Blood samples $(20 \mu \mathrm{l})$ were collected via a small nick in the tail vein at 120,150, 160, 170 and $180 \mathrm{~min}$ for the determination of plasma glucose and plasma $\left[{ }^{3} \mathrm{H}\right]$ glucose concentrations. An additional $10 \mu \mathrm{l}$ of blood were collected at 120 and $180 \mathrm{~min}$ to measure plasma insulin concentrations by RIA (Millipore, St. Charles, MO, USA). The concentrations of glucose in the plasma were analyzed via the glucose oxidase method (YSI 2700 Select, Yellow Springs Instruments, Yellow
Springs, OH, USA). The determination of plasma $\left[3-{ }^{3} \mathrm{H}\right]$ glucose was performed as described previously [38]. The appearance rates for glucose were calculated as the ratio of the $\left[3{ }^{3} \mathrm{H}\right]$ glucose infusion rate $(\mathrm{dpm} / \mathrm{min})$ to the specific activity of the plasma glucose $(\mathrm{dpm} / \mu \mathrm{mol})$. Data are presented as average values during the last $30 \mathrm{~min}$ of the experiment.

\section{Glucose production in primary hepatocytes}

Glucose production was determined by modified the protocol described by Foretz $\mathrm{M}$ et al. [39]. Briefly, primary mouse hepatocytes were isolated and plated in a 6-well collagen I coated plate (Biocoat plate, BD Biosciences, Bedford, MA) in DMEM containing antibiotics and $10 \%$ FBS for $4 \mathrm{~h}$, then switched to serum free DMEM with $100 \mathrm{nM}$ dexamethasone (Dex) for $16 \mathrm{~h}$ prior to the measurement of glucose production and washed once with PBS. Cells were then incubated in glucose-free DMEM containing $10 \mathrm{mM}$ lactate and $1 \mathrm{mM}$ pyruvate with $100 \mathrm{uM} \mathrm{Bt}_{2}$-cAMP (Sigma Aldrich, St. Louis, MO), with or without IL-22, AKT-inhibitor, or compound C (EMD Chemicals Inc. Gibbstown, NJ). Glucose production was detected at $8 \mathrm{~h}$ incubation and measured by using 2300 STAT Plus Glucose Analyzer (YSI Life Sciences, Yellow Springs, $\mathrm{OH})$. The value was normalized to the protein concentration.

\section{RNA extraction and real time quantitative RT-PCR}

RNA was extracted from the liver tissue with Trizol (Invitrogen, Calsbad, CA) or QIAGEN RNAeasy kit (QIAGEN, Valencia, CA) according to the manufacturer's instructions. mRNA expression of gluconeogenic genes was determined by real-time quantitative PCR, using a model 7500 PCR system (Applied Biosystems, Foster City, CA). Primers used in real-time PCR were described previously [39].

\section{Western blot analysis}

Liver tissue was homogenized in the RIPA Lysis buffer containing proteinase cocktail, PMSF, and sodium orthovanadate (Santa Cruz Biotechnology, Inc. Santa Cruz, CA) and grinded by Precellys 24 (Bertin Technologies, France). To isolate protein extracts from primary hepatocytes, cells were washed twice with iced cold PBS, mixed with RIPA Lysis buffer, and then sonicated on ice for $20 \mathrm{~s}$ prior to collecting protein solution. Western blot analyses were performed and protein bands were visualized by enhanced chemiluminescence reaction (Amersham Pharmacia Biotech, Piscataway, NJ).

\section{Blood chemistry}

Serum ALT levels were measured using chemistry analyzer (IDEXX Catalyst Dx, IDEXX Laboratories, Westbrook, ME). Serum insulin levels were determined using an ELISA 
kit (ALPCO Diagnostics, Salem, NH). Serum IL-22 levels were measured by an ELISA kit (R\&D system).

\section{Hepatic triglyceride contents}

Chloroform/methanol (2:1) solution was used for lipid extraction from the total liver. Extracted lipid was then dissolved in $5 \%$ triton X-100 solution and hepatic triglyceride levels was measured using EnzyChrom ${ }^{\mathrm{Ts}}$ triglyceride assay kit (BioAssay Systems, Hayward, CA).

\section{Histopathology}

For general histological analysis, liver tissues were fixed in the $10 \%$ neutralized formalin solution and embedded in the paraffin. Tissues were cut 4-um thickness and stained with hematoxylin and eosin $(\mathrm{H} \& \mathrm{E})$. For Oil red O staining for fat accumulation, frozen liver tissues were cut for $10 \mu \mathrm{m}$ sections with cryostat and stained with pre-warmed Oil Red O solution (Vector Laboratories, Burlingame, CA) for $10 \mathrm{~min}$, rinsed in water, and then counterstained with Mayer's hematoxylin, and analyzed by light microscopy.

\section{Administration of mice with IL-22 adenovirus}

IL-22 adenovirus was made by cloning mouse IL-22 cDNA (544 bp) into the pENTR/D-TOPO system (Invitrogen), followed by using Invitrogen Gateway system to perform a LR reaction with $\mathrm{pAd} / \mathrm{CMV} / \mathrm{V} 5-\mathrm{DEST}$ to make the expression vector $\mathrm{pAd} / \mathrm{CMV} / \mathrm{mIL}-22$. Mice were injected (intravenously) with adenovirus-IL-22 $\left(2 \times 10^{8} \mathrm{pfu}\right)$ or adenovirus-empty vector $\left(2 \times 10^{8} \mathrm{pfu}\right)$.

\section{Statistical analysis}

Data are expressed as the mean \pm SEM. To compare values obtained from two groups, Student's $t$-test was performed. To compare values obtained from three or more groups, one-way ANOVA was performed followed by Tukey's post-hoc test. A value of $P<0.05$ was considered significant.

\section{Abbreviations \\ Adeno-IL-22: IL-22 adenovirus; Adeno-vector: Control vector adenovirus; AMPK: Adenosine monophosphate-activated protein kinase; G6Pase: Glucose-6- phospatase; GTT: Glucose tolerance test; IL-22: Interleukin-22; ITT: Insulin tolerance test; PEPCK: Phosphoenolpyruvate carboxykinase; PGC-1a: Peroxisome proliferator activated receptor gamma coactivator 1-alpha; PI3 Kinase: Phospoinositide 3-kinase; PTT: Pyruvate tolerance test; TG: Transgenic; STAT3: signal transducer and activator of transcription 3; STAT3 ${ }^{\mathrm{Hep}-/-}$ mice: Hepatocyte-specific STAT3 knockout mice.}

\section{Competing interests}

The authors declare that they have no competing interests.

\section{Authors' contributions}

OP carried out the experiments, analyzed the data and participated in writing the paper. SHK, MX, HW, DF, JT, and DO carried out some experiments and analyzed the data. GK analyzed the data, wrote and edited the paper. BG conceived and directed the study and wrote the paper. All authors read and approved the final manuscript.

\section{Acknowledgment}

The authors greatly appreciated Oksana Gavrilova (NIDDK, NIH) for helping some glucose metabolism experiments.

\section{Author details}

'Laboratory of Liver Diseases, NIAAA/NIH, 5625 Fishers Lane, Bethesda, MD 20892, USA. ${ }^{2}$ Laboratory of Physiologic Studies, National Institute on Alcohol Abuse and Alcoholism, National Institutes of Health, Bethesda, MD 20892, USA. ${ }^{3}$ Laboratory of Toxicology, College of Pharmacy, Chosun University, Gwangju, South Korea. ${ }^{4}$ Obesity and Metabolism Laboratory, The Institute for Drug Research, School of Pharmacy, Faculty of Medicine, The Hebrew University of Jerusalem, Jerusalem 91120, Israel.

Received: 24 March 2015 Accepted: 14 May 2015

Published online: 30 May 2015

\section{References}

1. Ouyang W, Kolls JK, Zheng Y. The biological functions of Thelper 17 cell effector cytokines in inflammation. Immunity. 2008;28:454-67.

2. Sonnenberg GF, Fouser LA, Artis D. Border patrol: regulation of immunity, inflammation and tissue homeostasis at barrier surfaces by IL-22. Nat Immunol. 2011;12:383-90.

3. Wolk K, Witte E, Witte K, Warszawska K, Sabat R. Biology of interleukin-22. Semin Immunopathol. 2010;32:17-31.

4. Sabat R, Ouyang W, Wolk K. Therapeutic opportunities of the IL-22-IL-22R1 system. Nat Rev Drug Discov. 2014;13:21-38.

5. Rutz S, Eidenschenk C, Ouyang W. IL-22, not simply a Th17 cytokine. Immunol Rev. 2013;252:116-32.

6. Zenewicz LA, Flavell RA. Recent advances in IL-22 biology. Int Immunol. 2011:23:159-63.

7. Kong X, Feng D, Wang H, Hong F, Bertola A, Wang FS, et al. Interleukin-22 induces hepatic stellate cell senescence and restricts liver fibrosis in mice. Hepatology. 2012;56:1150-9.

8. Radaeva S, Sun R, Pan HN, Hong F, Gao B. Interleukin 22 (IL-22) plays a protective role in T cell-mediated murine hepatitis: IL-22 is a survival factor for hepatocytes via STAT3 activation. Hepatology. 2004;39:1332-42.

9. Zenewicz LA, Yancopoulos GD, Valenzuela DM, Murphy AJ, Karow M, Flavell RA. Interleukin-22 but not interleukin-17 provides protection to hepatocytes during acute liver inflammation. Immunity. 2007;27:647-59.

10. Ki SH, Park O, Zheng M, Morales-lbanez O, Kolls JK, Bataller R, et al. Interleukin-22 treatment ameliorates alcoholic liver injury in a murine model of chronic-binge ethanol feeding: role of signal transducer and activator of transcription 3. Hepatology. 2010;52:1291-300.

11. Muhl H, Scheiermann P, Bachmann M, Hardle L, Heinrichs A, Pfeilschifter J. IL-22 in tissue-protective therapy. Br J Pharmacol. 2013;169:761-71.

12. Sertorio M, Hou X, Carmo RF, Dessein H, Cabantous S, Abdelwahed M, et al. Interleukin-22 and IL-22 binding protein (IL-22BP) regulate fibrosis and cirrhosis in hepatitis $C$ virus and schistosome infections. Hepatology. 2015;61:1321-31.

13. Rao R, Graffeo CS, Gulati R, Jamal M, Narayan S, Zambirinis CP, et al. Interleukin 17-producing gammadeltaT cells promote hepatic regeneration in mice. Gastroenterology. 2014;147:473-84.

14. Park O, Wang H, Weng H, Feigenbaum L, Li H, Yin S, et al. In vivo consequences of liver-specific interleukin-22 expression in mice: Implications for human liver disease progression. Hepatology. 2011;54:252-61.

15. Waidmann O, Kronenberger B, Scheiermann P, Koberle V, Muhl H, Piiper A. Interleukin-22 serum levels are a negative prognostic indicator in patients with hepatocellular carcinoma. Hepatology. 2014;59:1207.

16. Jiang R, Tan Z, Deng L, Chen Y, Xia Y, Gao Y, et al. Interleukin-22 promotes human hepatocellular carcinoma by activation of STAT3. Hepatology. 2011;54:900-9.

17. Zhao J, Zhang Z, Luan Y, Zou Z, Sun Y, Li Y, et al. Pathological functions of interleukin-22 in chronic liver inflammation and fibrosis with hepatitis B virus infection by promoting T helper 17 cell recruitment. Hepatology. 2014;59:1331-42.

18. Liang SC, Nickerson-Nutter C, Pittman DD, Carrier Y, Goodwin DG, Shields KM, et al. IL-22 induces an acute-phase response. J Immunol. 2010;185:5531-8.

19. Wang X, Ota N, Manzanillo P, Kates L, Zavala-Solorio J, Eidenschenk C, et al. Interleukin-22 alleviates metabolic disorders and restores mucosal immunity in diabetes. Nature. 2014;514:237-41. 
20. Hasnain SZ, Borg DJ, Harcourt BE, Tong H, Sheng YH, Ng CP, et al. Glycemic control in diabetes is restored by therapeutic manipulation of cytokines that regulate beta cell stress. Nat Med. 2014;20:1417-26.

21. Yang L, Zhang Y, Wang L, Fan F, Zhu L, Li Z, et al. Amelioration of high fat diet induced liver lipogenesis and hepatic steatosis by interleukin-22. J Hepatol. 2010;53:339-47.

22. Upadhyay V, Poroyko V, Kim TJ, Devkota S, Fu S, Liu D, et al. Lymphotoxin regulates commensal responses to enable diet-induced obesity. Nat Immunol. 2012;13:947-53.

23. Dalmas E, Venteclef N, Caer C, Poitou C, Cremer I, Aron-Wisnewsky J, et al. T cell-derived IL-22 amplifies IL-1 beta-driven inflammation in human adipose tissue: relevance to obesity and type 2 diabetes. Diabetes. 2014;63:1966-77.

24. Dalmas E, Donath MY. A role for interleukin-22 in the alleviation of metabolic syndrome. Nat Med. 2014;20:1379-81.

25. Jiang W, Su J, Zhang X, Cheng X, Zhou J, Shi R, et al. Elevated levels of Th17 cells and Th17-related cytokines are associated with disease activity in patients with inflammatory bowel disease. Inflamm Res. 2014;63:943-50.

26. Shimauchi T, Hirakawa S, Suzuki T, Yasuma A, Majima Y, Tatsuno K, et al. Serum interleukin-22 and vascular endothelial growth factor serve as sensitive biomarkers but not as predictors of therapeutic response to biologics in patients with psoriasis. J Dermatol. 2013;40:805-12.

27. Fabbrini E, Cella M, McCartney SA, Fuchs A, Abumrad NA, Pietka TA, et al. Association between specific adipose tissue CD4+ T-cell populations and insulin resistance in obese individuals. Gastroenterology. 2013;145:366-74. e361-363.

28. Petersen PS, Lei X, Seldin MM, Rodriguez S, Byerly MS, Wolfe A, et al. Dynamic and extensive metabolic state-dependent regulation of cytokine expression and circulating levels. Am J Physiol Regul Integr Comp Physiol. 2014;307:R1458-70.

29. Feng $D$, Wang $Y$, Wang $H$, Weng $H$, Kong $X$, Martin-Murphy BV, et al. Acute and chronic effects of IL-22 on acetaminophen-induced liver injury. J Immunol. 2014;193:2512-8.

30. Feng D, Park O, Radaeva S, Wang H, Yin S, Kong X, et al. Interleukin-22 ameliorates cerulein-induced pancreatitis in mice by inhibiting the autophagic pathway. Int J Biol Sci. 2012;8:249-57.

31. Xue J, Nguyen DT, Habtezion A. Aryl hydrocarbon receptor regulates pancreatic IL-22 production and protects mice from acute pancreatitis. Gastroenterology. 2012;143:1670-80.

32. Sabat R. IL-10 family of cytokines. Cytokine Growth Factor Rev. 2010;21:315-24.

33. Matthys P, Billiau A. Cytokines and cachexia. Nutrition. 1997;13:763-70.

34. Inoue $\mathrm{H}$, Ogawa W, Ozaki M, Haga S, Matsumoto M, Furukawa K, et al. Role of STAT-3 in regulation of hepatic gluconeogenic genes and carbohydrate metabolism in vivo. Nat Med. 2004;10:168-74.

35. Taniguchi CM, Kondo T, Sajan M, Luo J, Bronson R, Asano T, et al. Divergent regulation of hepatic glucose and lipid metabolism by phosphoinositide 3-kinase via Akt and PKClambda/zeta. Cell Metab. 2006;3:343-53.

36. Zhang BB, Zhou G, Li C. AMPK: an emerging drug target for diabetes and the metabolic syndrome. Cell Metab. 2009;9:407-16.

37. MacLeod JN, Shapiro BH. Repetitive blood sampling in unrestrained and unstressed mice using a chronic indwelling right atrial catheterization apparatus. Lab Anim Sci. 1988;38:603-8.

38. Youn JH, Kim JK, Buchanan TA. Time courses of changes in hepatic and skeletal muscle insulin action and GLUT4 protein in skeletal muscle after STZ injection. Diabetes. 1994;43:564-71.

39. Foretz M, Hebrard S, Leclerc J, Zarrinpashneh E, Soty M, Mithieux G, et al. Metformin inhibits hepatic gluconeogenesis in mice independently of the LKB1/AMPK pathway via a decrease in hepatic energy state. J Clin Invest. 2010;120:2355-69.

\section{Submit your next manuscript to BioMed Central and take full advantage of:}

- Convenient online submission

- Thorough peer review

- No space constraints or color figure charges

- Immediate publication on acceptance

- Inclusion in PubMed, CAS, Scopus and Google Scholar

- Research which is freely available for redistribution 